\title{
Pre-vaccination immune response to COVID-19
}

\author{
Rujittika Mungmunpuntipantip ${ }^{1} \cdot$ Viroj Wiwanitkit $^{2}$
}

Received: 18 October 2021 / Accepted: 20 October 2021 / Published online: 28 October 2021

(c) The Author(s), under exclusive licence to Royal Academy of Medicine in Ireland 2021

\section{Dear Editor,}

We would like to share ideas on the publication "Prevaccination immune response to COVID-19 in a population in Northeast Portugal [1]." Duro et al. concluded that "IgM and IgG displayed a similar initial increase ... the identification of previously undiagnosed participants. [1]." We agree that the pre-vaccination antibody study might be useful. A diagnosis of previous asymptomatic infection can help gather correct epidemiological data. Additionally, the investigation might be useful for determining vaccine efficacy and surveillance of safety of vaccination. In a previous report, immune response to COVID-19 vaccine differs in cases with and without previous COVID-19 [2]. Additionally, a COVID-19 vaccine recipient with previous COVID-19 has a higher blood viscosity after vaccination, which might lead to hyperviscosity-associated adverse effect of vaccination [2].

\section{Declarations}

Conflict of interest The authors declare no competing interests.

\section{References}

1. Duro M, Duro I, Rebelo I et al (2021) Pre-vaccination immune response to COVID-19 in a population in Northeast Portugal. Ir J Med Sci https://doi.org/10.1007/s11845-021-02799-6. Online ahead of print

2. Joob B, Wiwanitkit V (2021) Viscosity after COVID-19 vaccination, hyperviscosity and previous COVID-19. Clin Appl Thromb Hemost Jan-Dec 27:10760296211020833

Publisher's Note Springer Nature remains neutral with regard to jurisdictional claims in published maps and institutional affiliations.
Rujittika Mungmunpuntipantip

rujittika@gmail.com

Private Academic Consultant, Bangkok, Thailand

2 Dr DY Patil University, Pune, India 